\title{
Adaptive Synchronization of Nonlinearly Parameterized Complex Dynamical Networks with Unknown Time-Varying Parameters
}

\author{
Tengfei Wang, Junmin Li, and Shu Tang \\ School of Science, Xidian University, Xi'an 710071, China \\ Correspondence should be addressed to Junmin Li, jmli@mail.xidian.edu.cn
}

Received 14 December 2011; Accepted 16 January 2012

Academic Editor: Teh-Lu Liao

Copyright (c) 2012 Tengfei Wang et al. This is an open access article distributed under the Creative Commons Attribution License, which permits unrestricted use, distribution, and reproduction in any medium, provided the original work is properly cited.

A new adaptive learning control approach is proposed for a class of nonlinearly parameterized complex dynamical networks with unknown time-varying parameters. By using the parameter separation and reparameterization technique, the adaptive learning laws of periodically timevarying and constant parameters and an adaptive control strategy are designed to ensure the asymptotic convergence of the synchronization error in the sense of square error norm. Then, a sufficient condition of the synchronization is given by constructing a composite energy function. Finally, an example of the complex network is used to verify the effectiveness of proposed approach.

\section{Introduction}

Since small world network and scale-free network were discovered in the past decade, complex dynamical networks have become a focus issue and have been studied intensively in various disciplines, such as sociology, biology, mathematics, and engineering [1,2]. As nonlinear dynamics and topological structure of the complex dynamical networks influence its dynamical behaviors, and synchronization is one of the basic forms of cooperative behavior, many of the basic mechanisms have a direct relationship with the synchronization. Research on synchronization of networks has an important significance on understanding of cooperative behavior mechanism and complex phenomena in nature and society. What is more is that it also has a very broad application prospects on nuclear magnetic resonance, wireless sensor network, and multirobot coordination. On the other hand, it can also provide the necessary theoretical basis simultaneously in multiple mobile robot systems, unmanned aircraft systems and distributed sensor array in the field of intelligent autonomous coordination control of complex systems. Up to now, synchronization can be roughly divided into complete 
synchronization and generalized synchronization. Generalized synchronization includes cluster synchronization, partial synchronization, and functional project synchronization.

However, there are many of real-world network systems such as biological networks, mobile communications networks, and social networks, whose structure will change over time, coupling parameter, and other system parameters. For example, the feed-forward loops (FFLs) are typical network motifs in many real world biological networks. The structures, functions, as well as noise characteristics of FFLs are dependent on the network parameters. In [3], a global relative parameter sensitivities (GRPSs) were proposed for FFLs in the genetic networks. The proposed GRPS approach sheds some light on the potential real world applications, such as the synthetic genetic circuits, predicting the effect of interventions in medicine and biotechnology, and so on. The existence of time-varying coupling parameter increases the difficulty of researching the problem of synchronization for complex networks. In the existing results [4-19], the method of synchronization is divided into two categories which are adaptive coupling and adaptive control approach. [20] studied a time-varying complex dynamical network model and its controlled synchronization criteria. It showed that synchronization of such a time-varying dynamical network was completely determined by the inner-coupling matrix and by the eigenvalues and the corresponding eigenvectors of the coupling configuration matrix of the network. [21] investigated pinning adaptive synchronization of a general complex dynamical network and indeed gave the positive answers to two questions which were how many nodes and how much coupling strength should a network with fixed structure and coupling be chosen. However, in [20, 21], they considered the controlled complex dynamical network with linearly diffusive couplings; [22] researched adaptive synchronization of an uncertain complex dynamical network. Although it considered complex dynamical network with uncertain nonlinear diffusive couplings, the uncertainty of the system with unknown time-varying parameters was not considered. [23] discussed a global payoff-based strategy updating model for studying cooperative behavior of a networked population. It characterized the interactions among individuals by time varying parameter. It is obvious that the coupling of actual complex networks is mostly complex, and the coupling parameters of the network may be not sure or change with some varying factors. Among the above results, none of them is concerning with the adaptive synchronization of complex network systems with unknown time-varying nonlinear coupling.

On the other hand, in recent years, adaptive learning control [24], a new adaptive control scheme, has been proposed to deal with the unknown periodic time-varying parameters. It used various methods to estimate the unknown constant or time-varying parameters of the system in order to obtain control input. The convergence of tracking error was proved by using the composite energy function. It is worth mentioning that learning control based on the composite energy function plays an important role in dealing with the estimates of periodically time-varying parameters.

Motivated by the above observations, in this paper, the problem of adaptive synchronization is investigated for nonlinearly parameterized complex dynamical networks with unknown time-varying parameters via adaptive learning control method. The main contribution of the paper is that, for the first time, the problem of adaptive synchronization for nonlinearly parameterized complex dynamical networks with unknown time-varying parameters is solved. By combining the parameter separation $[25,26]$ and reparameterization technique, the adaptive learning laws of periodically time-varying and constant parameters and the proposed adaptive controllers guarantee the asymptotic convergence of the synchronization error in the $L_{T}^{2}$ norm and that all closed-loop signals are bounded in the $L_{T}^{2}$ norm. Simulation results are provided to show the effectiveness of the proposed approach. 


\section{Problem Formulation and Preliminaries}

In this section, we introduce the network model considered in this paper and give some useful mathematical preliminaries.

Consider a dynamical network consisting of $N$ identical nodes with nonlinear couplings, in which each node is an $n$-dimensional dynamical system. The state equations of the network are

$$
\dot{x}_{i}(t)=f\left(x_{i}(t)\right)+\sum_{j=1}^{N} a_{i j} \Gamma g\left(x_{j}(t), \varphi_{i}(t)\right) \quad i=1,2 \ldots, N
$$

where $x_{i}(t)=\left[x_{i 1}(t), x_{i 2}(t), \ldots, x_{i n}(t)\right]^{\mathrm{T}} \in R^{n}$ represents the state vector of the $i$ th node. $f$ : $R^{n} \times R \rightarrow R^{n}$ is a smooth nonlinear vector-valued function. $\varphi_{i}(t)$ represents the unknown time-varying function. $g: R^{n} \times R \times R \rightarrow R^{n}$ is an unknown continuous nonlinear vectorvalued function. $a_{i j}=a_{j i}$ is the coupling element. If there exists a connection between node $i$ and node $j(i \neq j)$, then $a_{i j}=a_{j i}>0$, else $a_{i j}=a_{j i}=0$. The inner coupling matrix $\Gamma=$ $\operatorname{diag}\left(\gamma_{1}, \ldots, \gamma_{n}\right)$ is nonnegative definite.

Let

$$
A=\left(\begin{array}{cccc}
a_{11} & a_{12} & \cdots & a_{1 N} \\
a_{21} & a_{22} & \cdots & a_{2 N} \\
\vdots & \vdots & \ddots & \vdots \\
a_{N 1} & a_{N 2} & \cdots & a_{N N}
\end{array}\right)
$$

where $A^{\mathrm{T}}=A$ is an unknown irreducible real symmetric matrix. In this model, it is only required that the elements of the coupling matrix $A$ satisfy

$$
a_{i i}=-\sum_{j=1, j \neq i}^{N} a_{i j}<0, \quad i, j=1,2, \ldots, N \text {. }
$$

The system (2.1) is said to be asymptotic synchronization in $L_{T}^{2}$ norm if

$$
\lim _{t \rightarrow \infty} \int_{t-T}^{t}\left\|x_{i}(\tau)-s(\tau)\right\|^{2} d \tau=0, \quad i=1,2, \ldots, N
$$

where $\|\cdot\|$ stands for the Euclidean vector norm. Synchronous evolution $s(t)$ is an arbitrary desired state, which is also an isolated node of the network (2.1) with $\dot{s}(t)=f(s(t))$. We assume that for the system $\dot{s}(t)=f(s(t))$ there exists stable equilibrium point, stable periodic orbit, or even chaotic attractor.

Remark 2.1. It is well known that multiagent system is a special kind of complex networks. The consensus has similar meaning with synchronization, and consensus is a fundamental natural phenomenon in nature. [27] researched the cluster consensus of discrete-time multiagent systems. Based on Markov chains and nonnegative matrix analysis, two novel cluster consensus criteria were obtained for MAS with fixed and switching topology, 
respectively. However, in this paper, we consider a new synchronization problem for continuous-time complex dynamic network with nonlinearly time-varying coupling parameter. This problem is quite different from [27], and the challenge problem is due to the timevarying nonlinearly coupling parameter in (2.1).

Now, the following assumptions are introduced.

Assumption 2.2. In the network (2.1), suppose that there exists $l_{i}>0$, satisfying

$$
\left[x_{i}(t)-s(t)\right]^{T}\left[f\left(x_{i}(t)\right)-f(s(t))\right] \leq l_{i}\left[x_{i}(t)-s(t)\right]^{T}\left[x_{i}(t)-s(t)\right]
$$

where $x_{i}(t)$ and $s(t)$ are time varying vectors.

Lemma 2.3 (separation principle). For the unknown continuous function $g(\cdot, \cdot)$, the following inequality holds

$$
\left\|g\left(x_{j}(t), \varphi_{i}(t)\right)-g\left(s(t), \varphi_{i}(t)\right)\right\|^{2} \leq\left\|x_{j}(t)-s(t)\right\|^{2} h\left(x_{j}(t), s(t)\right) \lambda\left(\varphi_{i}(t)\right),
$$

where $h(\cdot, \cdot)$ is known nonnegative continuous function and $\lambda(\cdot)$ is unknown nonnegative continuous function.

Assumption 2.4. In the network $(2.1), \varphi_{i}(t)$ is unknown time-varying function with a known period $T$, in that way $\lambda\left(\varphi_{i}(t)\right)$ is also a periodic function. Suppose $\lambda\left(\varphi_{i}(t)\right)=\phi_{i}(t)+\theta_{i}$, where $\phi_{i}(t)$ is unknown continuous function and $\theta_{i}$ is unknown constant parameter.

Assumption 2.5. In the network (2.1), the inner coupling matrix $\Gamma$, coupling matrix $A$, and $h(\cdot, \cdot)$ satisfy

$$
\|\Gamma\|=\gamma, \quad h\left(x_{j}(t), s(t)\right)<H, \quad\left|a_{i j}\right| \leq a, \quad \forall i, j=1,2, \ldots, N,
$$

where $\gamma, H, a$ are positive constants.

Lemma 2.6 (Young's inequality). For any vectors $x, y \in R^{n}$, and any $c>0$, the following matrix inequality holds:

$$
x^{T} y \leq c x^{T} x+\frac{1}{4 c} y^{T} y
$$

\section{Adaptive Controller Design}

To achieve the control objective (2.4), we need an adaptive control strategy to nodes in the network (2.1). Then the controlled network is given by

$$
\dot{x}_{i}(t)=f\left(x_{i}(t)\right)+\sum_{j=1}^{N} a_{i j} \Gamma g\left(x_{j}(t), \varphi_{i}(t)\right)+u_{i}(t), \quad i=1,2, \ldots, N
$$


where $u_{i}(t)=\left[u_{i 1}(t), u_{i 2}(t), \ldots, u_{i n}(t)\right]^{\mathrm{T}} \in R^{n}, i=1,2, \ldots, N$ are the adaptive controllers to be designed. Let synchronization error be $e_{i}(t)=x_{i}(t)-s(t), i=1,2, \ldots, N$ and we have the following dynamical error equations:

$$
\dot{e}_{i}(t)=f\left(x_{i}(t)\right)-f(s(t))+\sum_{j=1}^{N} a_{i j} \Gamma\left(g\left(x_{j}(t), \varphi_{i}(t)\right)-g\left(s(t), \varphi_{i}(t)\right)\right)+u_{i}(t) .
$$

Then we design controllers by

$$
u_{i}(t)=-\operatorname{Nar} H\left(\widehat{\phi}_{i}(t)+\widehat{\theta}_{i}(t)\right)\left(x_{i}(t)-s(t)\right)
$$

where $\gamma, a, H$ satisfy condition (2.7), $N$ is the node number of the network (2.1), and $\widehat{\phi}_{i}(t), \widehat{\theta}_{i}(t)$ are estimations to $\phi_{i}(t), \theta_{i}$, respectively. For convenience, we denote

$$
\tilde{\phi}_{i}(t)=\phi_{i}(t)-\widehat{\phi}_{i}(t), \quad \tilde{\theta}_{i}(t)=\theta_{i}-\widehat{\theta}_{i}(t) .
$$

The constant parameter update law and the time-varying periodic adaptive learning law are designed as follows:

$$
\begin{gathered}
\dot{\hat{\theta}}_{i}(t)=r_{i} e_{i}^{\mathrm{T}}(t) e_{i}(t), \\
\widehat{\phi}_{i}(t)= \begin{cases}\widehat{\phi}_{i}(t-T)+q_{i} e_{i}^{\mathrm{T}}(t) e_{i}(t), & t \in[T,+\infty), \\
q_{i 0}(t) e_{i}^{\mathrm{T}}(t) e_{i}(t), & t \in[0, T), \\
0, & t \in[-T, 0),\end{cases}
\end{gathered}
$$

where $r_{i}, q_{i}>0$ are constants and $q_{i 0}(t)$ is a continuous and strictly increasing nonnegative function satisfying $q_{i 0}(0)=0, q_{i 0}(T)=q_{i}$, which ensure $\widehat{\phi}_{i}(t)$ is continuous in $t=i T, i=$ $1,2, \ldots, N$.

\section{Convergence Analysis}

In the section, we will give the convergence of the proposed algorithm in the following theorem.

Theorem 4.1. Under Assumptions 2.2-2.5, the control law (3.3) with update law (3.5) and the periodic adaptive law (3.6), guarantees the $L_{T}^{2}$ asymptotic synchronization of the controlled network (3.1), while keeping all closed-loop signals are $L_{T}^{2}$ bounded. 
Proof. To facilitate the convergence analysis, we choose a Lyapunov-Krasovskii function as follows:

$$
V(t)= \begin{cases}\frac{1}{2} \sum_{i=1}^{N} e_{i}^{\mathrm{T}} e_{i}+\frac{N \operatorname{ar} H}{2} \sum_{i=1}^{N} \int_{0}^{t} q_{i}^{-1} \tilde{\phi}_{i}^{2}(\tau) d \tau+\frac{N a \gamma H}{2} \sum_{i=1}^{N} r_{i}^{-1}\left(\tilde{\theta}_{i}(t)+L_{i}\right)^{2}, & t \in[0, T), \\ \frac{1}{2} \sum_{i=1}^{N} e_{i}^{\mathrm{T}} e_{i}+\frac{N \operatorname{ar} H}{2} \sum_{i=1}^{N} \int_{t-T}^{t} q_{i}^{-1} \tilde{\phi}_{i}^{2}(\tau) d \tau+\frac{N a \gamma H}{2} \sum_{i=1}^{N} r_{i}^{-1}\left(\tilde{\theta}_{i}(t)+L_{i}\right)^{2}, & t \in[T,+\infty),\end{cases}
$$

where $L_{i}$ is sufficiently large positive constant which will be determined.

Firstly, we will prove the finiteness of $V(t)$ in $[0, T)$. Secondly, we will prove the asymptotical convergence of $e_{i}(t)$. Finally, we will prove all closed-loop signals are $L_{T}^{2}$ bounded.

First step, let us confirm the finiteness property of $V(t)$ for the first period $[0, T)$. From the system dynamics (3.1) and the proposed control laws (3.3), (3.5), and (3.6), it can be seen that the right-hand side of (3.1) is continuous with respect to all arguments. In light of the existence theorem of differential equation, (3.2) has unique solution in an interval $\left[0, T_{1}\right) \subset$ $[0, T)$, with $0<T_{1} \leq T$. Therefore, the boundedness of $V(t)$ over $\left[0, T_{1}\right)$ can be guaranteed and we need only focus on the interval $\left[T_{1}, T\right)$.

For any $t \in\left[T_{1}, T\right)$, the time derivative of $V(t)$ for $t \in\left[T_{1}, T\right)$ is given by

$$
\dot{V}(t)=\sum_{i=1}^{N} e_{i}^{T}(t) \dot{e}_{i}(t)+\frac{N a \gamma H}{2} \sum_{i=1}^{N} q_{i}^{-1} \tilde{\phi}_{i}^{2}(t)+N a r H \sum_{i=1}^{N} r_{i}^{-1}\left(\widetilde{\theta}_{i}(t)+L_{i}\right) \dot{\tilde{\theta}}_{i}(t), \quad t \in\left[T_{1}, T\right) .
$$

Taking the first term on the right-hand side of (4.2), one obtains

$$
\sum_{i=1}^{N} e_{i}^{\mathrm{T}}(t) \dot{e}_{i}(t)=\sum_{i=1}^{N} e_{i}^{\mathrm{T}}(t)\left[f\left(x_{i}(t)\right)-f(s(t))+\sum_{j=1}^{N} a_{i j} \Gamma\left(g\left(x_{j}(t), \varphi_{i}(t)\right)-g\left(s(t), \varphi_{i}(t)\right)\right)+u_{i}(t)\right] .
$$

Let $g\left(x_{j}(t), \varphi_{i}(t)\right)-g\left(s(t), \varphi_{i}(t)\right)=\Lambda$, and

$$
\|\Lambda\|^{2} \leq\left\|e_{j}(t)\right\|^{2} h\left(x_{j}(t), s(t)\right) \lambda\left(\varphi_{i}(t)\right) .
$$


Then according to Assumption 2.2 and Lemma 2.6, we get

$$
\begin{aligned}
\sum_{i=1}^{N} e_{i}^{\mathrm{T}}(t) \dot{e}_{i}(t) & \leq \sum_{i=1}^{N}\left[l_{i} e_{i}^{\mathrm{T}} e_{i}+\sum_{j=1}^{N} a_{i j} e_{i}^{\mathrm{T}} \Gamma \Lambda-\operatorname{NarH}\left(\widehat{\phi}_{i}(t)+\widehat{\theta}_{i}(t)\right) e_{i}^{\mathrm{T}} e_{i}\right] \\
& \leq \sum_{i=1}^{N}\left[l_{i} e_{i}^{\mathrm{T}} e_{i}+\sum_{j=1}^{N}\left(a_{i j}^{2} c e_{i}^{\mathrm{T}} e_{i}+\frac{1}{4 c} \Lambda^{\mathrm{T}} \Gamma^{\mathrm{T}} \Gamma \Lambda\right)-N a \gamma H\left(\widehat{\phi}_{i}(t)+\widehat{\theta}_{i}(t)\right) e_{i}^{\mathrm{T}} e_{i}\right] \\
& \leq \sum_{i=1}^{N}\left[l_{i} e_{i}^{\mathrm{T}} e_{i}+N a^{2} c e_{i}^{\mathrm{T}} e_{i}+\frac{N H \gamma^{2}}{4 c} e_{i}^{\mathrm{T}} e_{i}\left(\phi_{i}(t)+\theta_{i}\right)-\operatorname{Nar} H\left(\widehat{\phi}_{i}(t)+\widehat{\theta}_{i}(t)\right) e_{i}^{\mathrm{T}} e_{i}\right],
\end{aligned}
$$

where $c$ is a positive constant. If choosing $c=\gamma / 4 a$, we have

$$
\sum_{i=1}^{N} e_{i}^{\mathrm{T}}(t) \dot{e}_{i}(t) \leq \sum_{i=1}^{N}\left[l_{i}+\frac{N a \gamma}{4}+\operatorname{Na\gamma H}\left(\tilde{\phi}_{i}(t)+\tilde{\theta}_{i}(t)\right)\right] e_{i}^{\mathrm{T}} e_{i}
$$

Taking the third term on the right-hand side of (4.2), we have

$$
\operatorname{NarH} \sum_{i=1}^{N} r_{i}^{-1}\left(\widetilde{\theta}_{i}(t)+L_{i}\right) \dot{\tilde{\theta}}_{i}(t)=-N \operatorname{Nar} H \sum_{i=1}^{N}\left(\widetilde{\theta}_{i}(t)+L_{i}\right) e_{i}^{\mathrm{T}} e_{i} .
$$

Let us focus on the second term on the right-hand side of (4.2). Since $q_{i 0}(t)$ is a continuous function and strictly increasing in $[0, T), q_{i}^{-1} \leq q_{i 0}^{-1}(t)<\infty$ is ensured in the interval $\left[T_{1}, T\right)$, and

$$
\begin{aligned}
\frac{N \operatorname{ar} H}{2} \sum_{i=1}^{N} q_{i}^{-1} \tilde{\phi}_{i}^{2}(t) & \leq \frac{N \operatorname{arH}}{2} \sum_{i=1}^{N} q_{i 0}^{-1} \tilde{\phi}_{i}^{2}(t) \\
& =\frac{N \operatorname{ar} H}{2} \sum_{i=1}^{N} q_{i 0}^{-1}\left[\phi_{i}^{2}(t)+2 \widehat{\phi}_{i}^{2}(t)-2 \phi_{i}(t) \widehat{\phi}_{i}(t)-\widehat{\phi}_{i}^{2}(t)\right] \\
& \leq \frac{N a \gamma H}{2} \sum_{i=1}^{N} q_{i 0}^{-1}\left[\phi_{i}^{2}(t)+2 \widehat{\phi}_{i}^{2}(t)-2 \phi_{i}(t) \widehat{\phi}_{i}(t)\right] \\
& =\frac{N a r H}{2} \sum_{i=1}^{N} q_{i 0}^{-1} \phi_{i}^{2}(t)-N \operatorname{NarH} \sum_{i=1}^{N} \tilde{\phi}_{i}(t) e_{i}^{\mathrm{T}} e_{i} .
\end{aligned}
$$

Substituting (4.6)-(4.8) into (4.2) yields

$$
\dot{V}(t) \leq \sum_{i=1}^{N}\left(l_{i}+\frac{N a \gamma}{4}-N a \gamma H L_{i}\right) e_{i}^{\mathrm{T}} e_{i}+\frac{N a \gamma H}{2} \sum_{i=1}^{N} q_{i 0}^{-1}(t) \phi_{i}^{2}(t)
$$


It is obviously that there exists sufficiently large positive constants $L_{i}$ such that $l_{i}+$ $(\mathrm{Nar} / 4)-\mathrm{NarHL}_{i}<0$. According to (4.9), we have

$$
\dot{V}(t) \leq \frac{N a \gamma H}{2} \sum_{i=1}^{N} q_{i 0}^{-1}(t) \phi_{i}^{2}(t)
$$

Since $\phi_{i}(t)$ is continuous and periodic, its boundedness can be obtained. The boundedness of $\phi_{i}(t)$ leads to the boundedness of $\dot{V}(t)$. For $V\left(T_{1}\right)$ is bounded, the finiteness of $V(t)$ is obvious, for all $t \in\left[T_{1}, T\right)$.

Next step, we prove the asymptotical convergence of $e(t)$.

According to (4.1), we can get

$$
\begin{aligned}
\Delta V(t)= & V(t)-V(t-T) \\
= & \frac{1}{2} \sum_{i=1}^{N} e_{i}^{\mathrm{T}}(t) e_{i}(t)-\frac{1}{2} \sum_{i=1}^{N} e_{i}^{\mathrm{T}}(t-T) e_{i}(t-T)+\frac{N a \gamma H}{2} \sum_{i=1}^{N} \int_{t-T}^{t}\left[q_{i}^{-1} \tilde{\phi}_{i}^{2}(\tau)-q_{i}^{-1} \tilde{\phi}_{i}^{2}(\tau-T)\right] d \tau \\
& +\frac{N a \gamma H}{2} \sum_{i=1}^{N} r_{i}^{-1}\left(\tilde{\theta}_{i}(t)+L_{i}\right)^{2}-\frac{N a \gamma H}{2} \sum_{i=1}^{N} r_{i}^{-1}\left(\tilde{\theta}_{i}(t-T)+L_{i}\right)^{2} .
\end{aligned}
$$

Looking into the first two terms on the right-hand side of (4.11), with Newton-Leibniz formula, we obtain

$$
\begin{aligned}
\frac{1}{2} \sum_{i=1}^{N} e_{i}^{\mathrm{T}}(t) e_{i}(t)-\frac{1}{2} \sum_{i=1}^{N} e_{i}^{\mathrm{T}}(t-T) e_{i}(t-T) & =\sum_{i=1}^{N} \int_{t-T}^{t} e_{i}^{\mathrm{T}}(\tau) \dot{e}_{i}(\tau) d \tau \\
& \leq \sum_{i=1}^{N} \int_{t-T}^{t}\left[l_{i}+\frac{N a \gamma}{4}+\operatorname{Nar} H\left(\tilde{\phi}_{i}+\tilde{\theta}_{i}\right)\right] e_{i}^{\mathrm{T}}(\tau) e_{i}(\tau) d \tau
\end{aligned}
$$

Using the algebraic relation

$$
(a-b)^{\mathrm{T}} H(a-b)-(a-c)^{\mathrm{T}} H(a-c)=(c-b)^{\mathrm{T}} H[2(a-b)+(b-c)],
$$

where $a, b, c \in R^{p}, H \in R^{p \times p}$, and taking the third term on the right-hand side of (4.11), one obtains

$$
\frac{N a \gamma H}{2} \sum_{i=1}^{N} \int_{t-T}^{t}\left[q_{i}^{-1} \tilde{\phi}_{i}^{2}(\tau)-q_{i}^{-1} \tilde{\phi}_{i}^{2}(\tau-T)\right] d \tau=-N a \gamma H \sum_{i=1}^{N} \int_{t-T}^{t}\left[\tilde{\phi}_{i}(\tau)+\frac{1}{2} q_{i} e_{i}^{\mathrm{T}} e_{i}\right] e_{i}^{\mathrm{T}} e_{i} d \tau
$$


The last two terms of right-hand side of (4.11) can be simplified as follows:

$$
\begin{gathered}
\frac{N a \gamma H}{2} \sum_{i=1}^{N} r_{i}^{-1}\left(\tilde{\theta}_{i}(t)+L_{i}\right)^{2}-\frac{N a \gamma H}{2} \sum_{i=1}^{N} r_{i}^{-1}\left(\tilde{\theta}_{i}(t-T)+L_{i}\right)^{2} \\
\quad=N a \gamma H \sum_{i=1}^{N} \int_{t-T}^{t} r_{i}^{-1}\left(\tilde{\theta}_{i}(\tau)+L_{i}\right) \dot{\tilde{\theta}}_{i}(\tau) d \tau \\
=-N a \gamma H \sum_{i=1}^{N} \int_{t-T}^{t}\left(\tilde{\theta}_{i}(\tau)+L_{i}\right) e_{i}^{\mathrm{T}}(\tau) e_{i}(\tau) d \tau .
\end{gathered}
$$

Substituting (4.12)-(4.15) into (4.11), we can attain

$$
\Delta V(t) \leq-\sum_{i=1}^{N} \int_{t-T}^{t}\left(N a \gamma H L_{i}-l_{i}-\frac{N a \gamma}{4}\right) e_{i}^{\mathrm{T}}(\tau) e_{i}(\tau) d \tau
$$

Choosing $L_{i}>\left(l_{i} / N a \gamma H\right)+(1 / 4 H)$, we can obtain

$$
\Delta V(t)<0
$$

Applying (4.16) repeatedly for any $t \in[l T,(l+1) T], l=1,2, \ldots$, and denoting $t_{0}=t-l T$, we have

$$
V(t)=V\left(t_{0}\right)+\sum_{j=0}^{l-1} \Delta V(t-j T)
$$

Considering $t_{0} \in[0, T)$ and the positive of $V(t)$, according to (4.18), we obtain

$$
\lim _{t \rightarrow \infty} V(t)<\max _{t_{0} \in[0, T)} V\left(t_{0}\right)-\lim _{l \rightarrow \infty} \sum_{j=0}^{l-1} \sum_{i=1}^{N} \int_{t-(j+1) T}^{t-j T}\left(N a \gamma H L_{i}-l_{i}-\frac{N a \gamma}{4}\right) e_{i}^{\mathrm{T}}(\tau) e_{i}(\tau) d \tau
$$

Since $V\left(t_{0}\right)$ is bounded in the interval $[0, T)$, according to the convergence theorem of the sum of series and (4.19), the error $e(t)$ converges to zero asymptotically in $L_{T}^{2}$ norm. That is to say, we have

$$
\lim _{t \rightarrow \infty} \int_{t-T}^{t} e_{i}^{\mathrm{T}}(\tau) e_{i}(\tau) d \tau=0
$$


Finally, we prove that all the closed-loop signals are bounded in $L_{T}^{2}$ norm sense. For all $t \in[T, \infty)$, the derivative of $V(t)$ is

$$
\begin{aligned}
\dot{V}(t)= & \sum_{i=1}^{N} e_{i}^{\mathrm{T}}(t) \dot{e}_{i}(t)+\frac{N a \gamma H}{2} \sum_{i=1}^{N} q_{i}^{-1} \tilde{\phi}_{i}^{2}(t)-\frac{N a \gamma H}{2} \sum_{i=1}^{N} q_{i}^{-1} \tilde{\phi}_{i}^{2}(t-T) \\
& +N a \gamma H \sum_{i=1}^{N} r_{i}^{-1}\left(\widetilde{\theta}_{i}(t)+L_{i}\right) \dot{\tilde{\theta}}_{i}(t) \\
\leq & -\frac{N a \gamma H}{2} \sum_{i=1}^{N} q_{i}\left(e_{i}^{\mathrm{T}} e_{i}\right)^{2}-\sum_{i=1}^{N}\left(N a \gamma H L_{i}-l_{i}-\frac{N a \gamma}{4}\right) e_{i}^{\mathrm{T}} e_{i} .
\end{aligned}
$$

By (4.21), one can obtain

$$
V(t) \leq V(T)-\frac{N a \gamma H}{2} \sum_{i=1}^{N} \int_{T}^{t} q_{i}\left(e_{i}^{\mathrm{T}}(\tau) e_{i}(\tau)\right)^{2} d \tau-\sum_{i=1}^{N} \int_{T}^{t}\left(N a \gamma H L_{i}-l_{i}-\frac{N a \gamma}{4}\right) e_{i}^{\mathrm{T}} e_{i} d \tau .
$$

Choosing $L_{i}>\left(l_{i} / \mathrm{Nar} H\right)+(1 / 4 H)$, we have

$$
V(t)<V(T) .
$$

From the boundedness of $V(t)$ and (4.1), we can conclude that $e_{i}, \int_{t-T}^{t} \tilde{\phi}_{i}^{2}(\tau) d \tau, \widetilde{\theta}_{i}(t)$ are all bounded. Since $\phi_{i}(t)$ is continuous periodic function, it implies the $L_{T}^{2}$ boundedness of $\widehat{\phi}_{i}(t)$ and $\widehat{\theta}_{i}(t)$. According to (3.3), the $L_{T}^{2}$ boundedness of the control input $u_{i}(t)$ is obtained. Since $e_{i}(t)$ is bounded, the boundedness of $x_{i}(t)$ is received. So the proof is completed.

\section{Simulation Examples}

To demonstrate the theoretical result obtained in Section 3, the Chuas chaotic circuit is used as a dynamical node of the network.

Consider the Chuas chaos circuit

$$
\begin{gathered}
\dot{x}_{1}=p\left(-x_{1}+x_{2}-g\left(x_{1}\right)\right), \\
\dot{x}_{2}=x_{1}-x_{2}+x_{3}, \\
\dot{x}_{3}=-q x_{2}
\end{gathered}
$$

where $g\left(x_{1}\right)=m_{0} x_{1}+(1 / 2)\left(m_{1}-m_{0}\right)\left(\left|x_{1}+1\right|-\left|x_{1}-1\right|\right)$ with $p=10, q=14.7, m_{0}=-0.68$ and $m_{1}=-1.27$.

We take the system (5.1) as identical nodes of the network, which is given by

$$
\left(\begin{array}{c}
\dot{x}_{i 1} \\
\dot{x}_{i 2} \\
\dot{x}_{i 3}
\end{array}\right)=\left(\begin{array}{c}
p\left(-x_{i 1}+x_{i 2}-g\left(x_{i 1}\right)\right) \\
x_{i 1}-x_{i 2}+x_{i 3} \\
-q x_{i 2}
\end{array}\right)+\sum_{j=1}^{N} a_{i j} \Gamma \exp \left(-\varphi_{i}(t)\left(\begin{array}{c}
x_{j 1}^{2}(t) \\
x_{j 2}^{2}(t) \\
x_{j 3}^{2}(t)
\end{array}\right)\right)+u_{i}(t), \quad i=1,2, \ldots, N,
$$


where

$$
A=\left(\begin{array}{cccccc}
-1 & 1 & 0 & 0 & 0 & 0 \\
1 & -2 & 1 & 0 & 0 & 0 \\
0 & 1 & -3 & 2 & 0 & 0 \\
0 & 0 & 2 & -4 & 1 & 1 \\
0 & 0 & 0 & 1 & -5 & 4 \\
0 & 0 & 0 & 1 & 4 & -5
\end{array}\right) \quad \Gamma=\left(\begin{array}{lll}
1 & 0 & 0 \\
0 & 1 & 0 \\
0 & 0 & 1
\end{array}\right)
$$

Nonlinearly parameterized function satisfies

$$
\left\|\exp \left(-\varphi_{i}(t)\left(\begin{array}{c}
x_{j 1}^{2}(t) \\
x_{j 2}^{2}(t) \\
x_{j 3}^{2}(t)
\end{array}\right)\right)-\exp \left(-\varphi_{i}(t)\left(\begin{array}{c}
s^{2}(t) \\
s^{2}(t) \\
s^{2}(t)
\end{array}\right)\right)\right\|^{2} \leq\left\|e_{j}(t)\right\|^{2} 2 \varphi_{i}(t) \exp (-1) .
$$

The parameters are selected as follows:

$$
\begin{gathered}
N=6, \quad T=6, \quad a=5, \quad r=1, \quad H=5, \\
\phi_{1}(t)=0.2 \sin \frac{2 \pi t}{3}, \quad \phi_{2}(t)=2 \cos \pi t, \quad \phi_{3}(t)=-\sin \frac{2 \pi t}{3}, \quad \phi_{4}(t)=\cos \pi t, \\
\phi_{5}(t)=-2 \sin \frac{2 \pi t}{3}, \quad \phi_{6}(t)=2 \sin \frac{2 \pi t}{3}, \quad \theta=(2,10,8,3,2,5)^{\mathrm{T}} .
\end{gathered}
$$

In the following simulations, we choose

$$
\begin{aligned}
& q_{1}=0.0001, \quad q_{2}=0.0003, \quad q_{3}=0.0002, \quad q_{4}=0.0005, \quad q_{5}=0.0001, \quad q_{6}=0.0002, \\
& q_{10}(t)=\frac{t}{6} q_{1}, \quad q_{20}(t)=\frac{t}{6} q_{2}, \quad q_{30}(t)=\frac{t}{6} q_{3}, \quad q_{40}(t)=\frac{t}{6} q_{4}, \quad q_{50}(t)=\frac{t}{6} q_{5}, \quad q_{60}(t)=\frac{t}{6} q_{6},
\end{aligned}
$$

$r_{1}=r_{2}=r_{3}=r_{4}=r_{5}=r_{6}=0.0005$. The initially estimated value of the unknown parameter $\phi(t)$ is $\widehat{\phi}(0)=(0,0,0,0,0,0)^{\mathrm{T}}$, and the initial states are chosen as

$$
\begin{gathered}
x_{1}(0)=(2,0,-1)^{\mathrm{T}}, \quad x_{2}(0)=(3,1,-2)^{\mathrm{T}}, \quad x_{3}(0)=(-3,-2,1)^{\mathrm{T}}, \quad x_{4}(0)=(-1,0,-2)^{\mathrm{T}}, \\
x_{5}(0)=(-1,1,0)^{\mathrm{T}}, \quad x_{6}(0)=(3,-2,0.8)^{\mathrm{T}}, \quad s(0)=(-0.2,0.2,0.5)^{\mathrm{T}} .
\end{gathered}
$$

According to Theorem 4.1, the complex dynamical network (5.2) can be synchronized by applying the adaptive controllers (3.3) and the adaptive learning laws of parameters (3.5) and (3.6). Figure 1 shows the error evolutions under the designed controllers. We clearly show that the states of the network (5.2) asymptotically synchronize in $L_{T}^{2}$ norm with the states of the desired orbit (5.1) showed in Figure 2. Figure 3 depicts the time evolution of the controllers, and Figure 4 shows the evolution of the estimated time-varying parameters. 


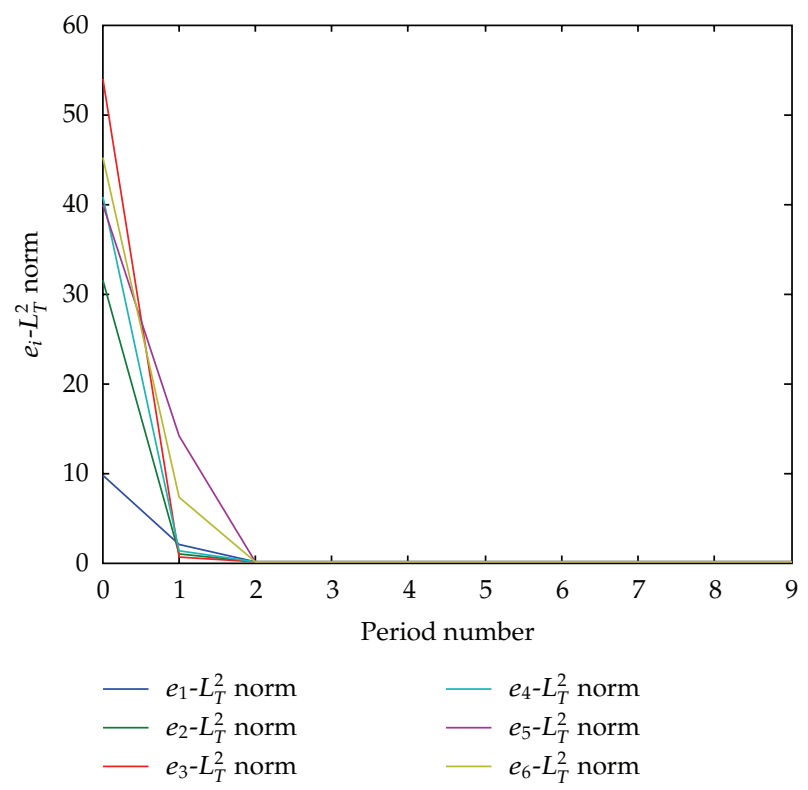

Figure 1: The synchronization errors in $L_{T}^{2}$ norm.

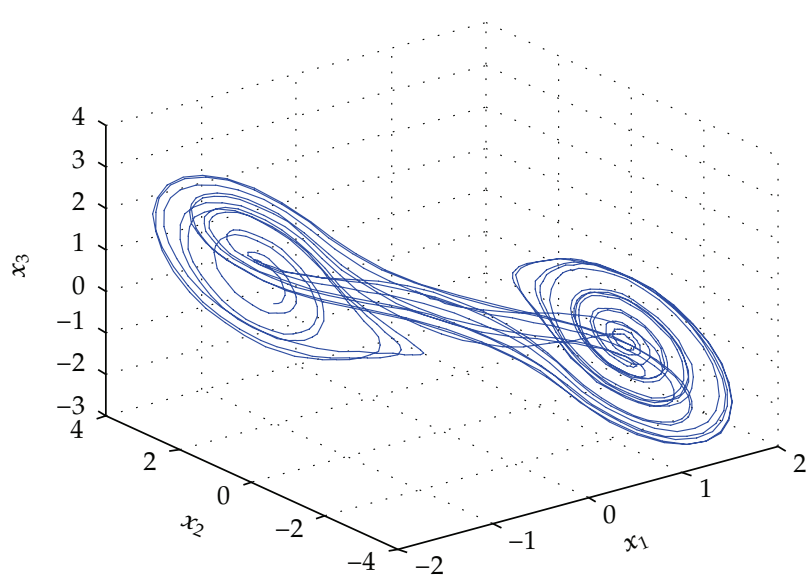

Figure 2: The desired orbit.

Figure 5 displays the evolution of the estimated constant parameters. Obviously, Figures 3-5 show that all the control signals and estimated parameters are also bounded.

\section{Conclusion}

In this paper, a new adaptive learning control method applied in nonlinearly parameterized complex dynamical networks with unknown time-varying parameters is presented. By 

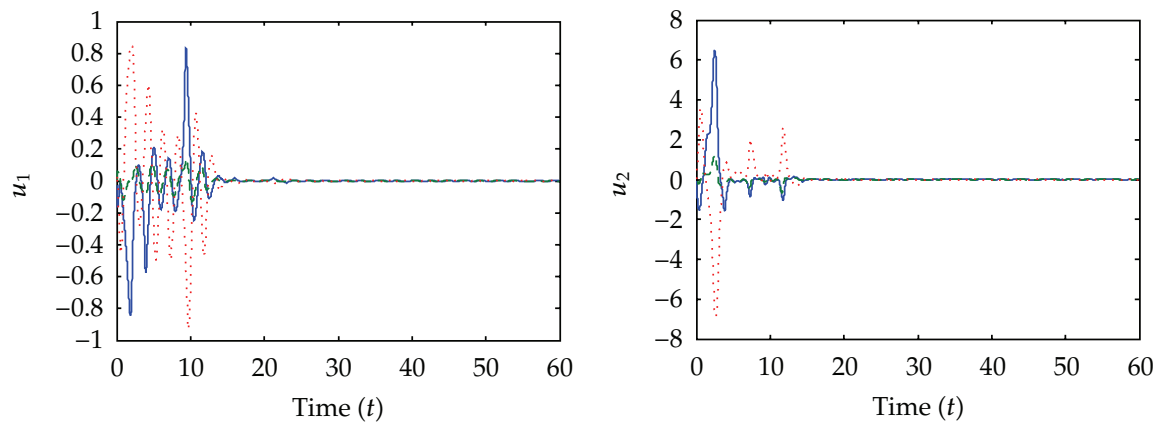

$$
\begin{aligned}
& -u_{11} \\
& ---u_{12}
\end{aligned}
$$

(a)

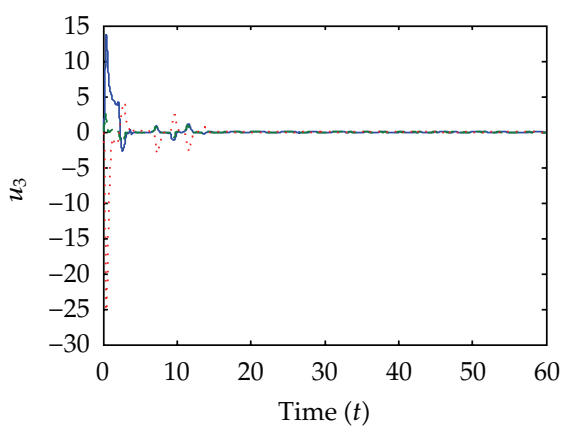

$-u_{31}$

- - $u_{32}$

… $u_{33}$

(c)

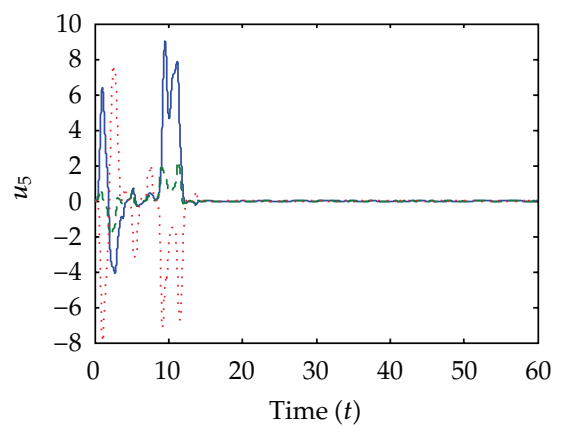

$-u_{51}$

$---u_{52}$

$\ldots u_{53}$ $-u_{21}$

$---u_{22}$

(b)

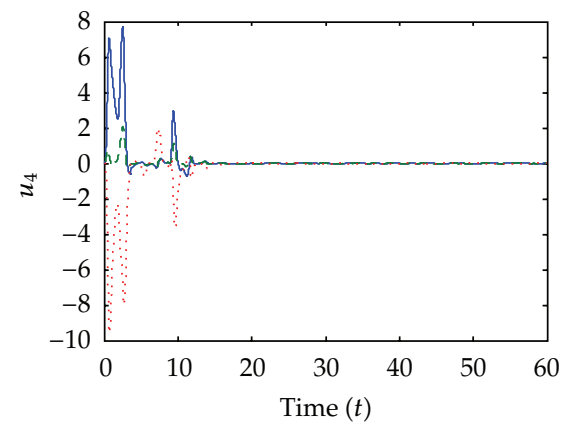

$-u_{41}$

$\begin{array}{ll}--- & u_{42} \\ \ldots \ldots & u_{43}\end{array}$

(d)

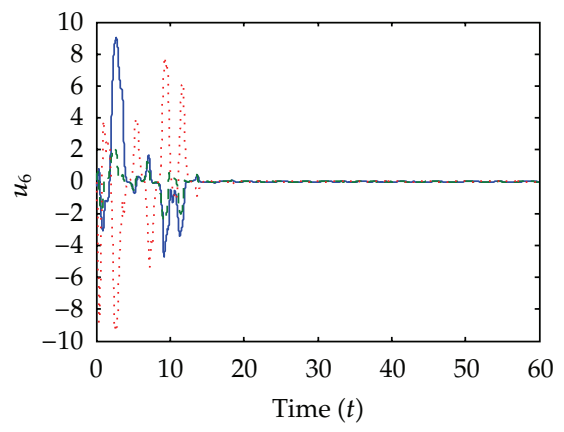

$-u_{61}$

…. $u_{63}$

(e)

(f)

Figure 3: The curve of control inputs $u_{i}$. 


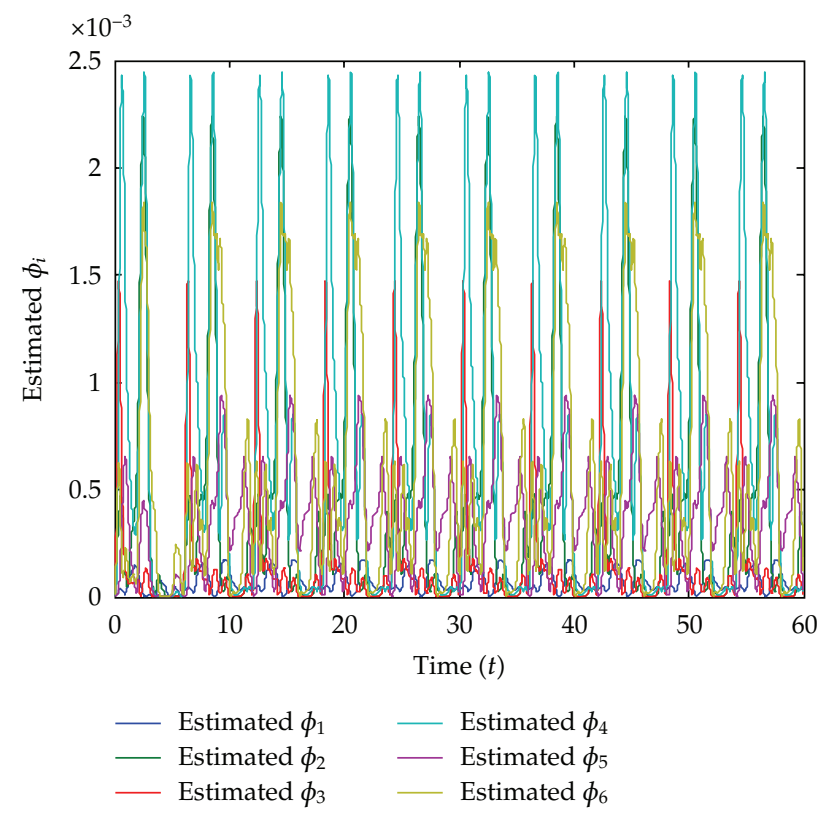

Figure 4: The evaluations of parameters $\phi_{i}$.

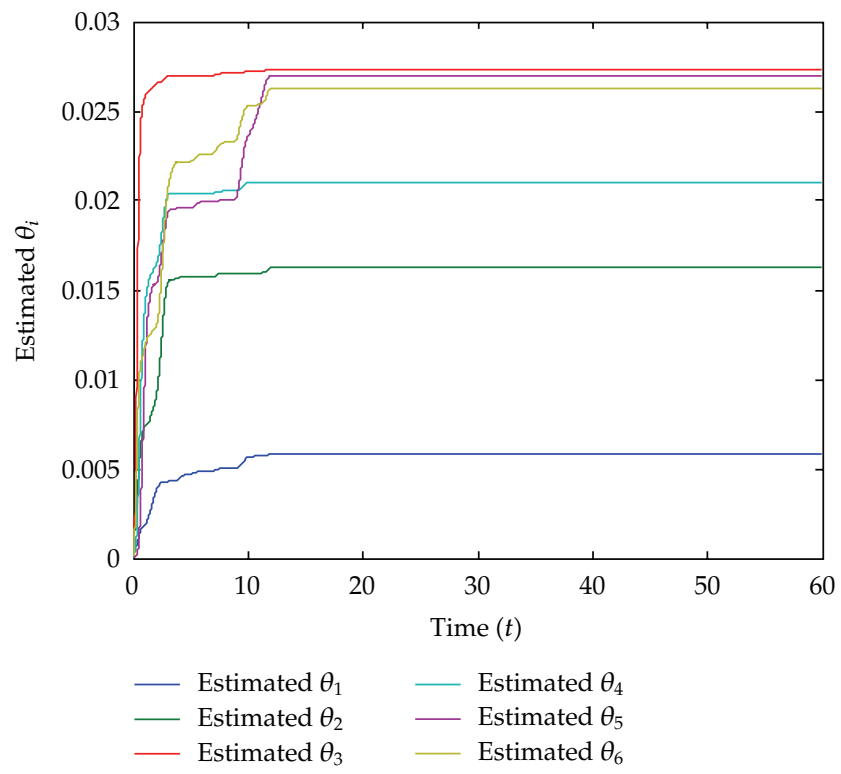

Figure 5: The evaluations of parameters $\theta_{i}$.

using the parameter separation technique and constructing a composite energy function, the adaptive learning laws of parameters and the adaptive control strategy are designed to ensure the asymptotic convergence of the synchronization error in the sense of square error norm. An example of the complex network is finally used to verify the proposed theoretical result. 


\section{Acknowledgments}

This work was supported by the National Natural Science Foundation of China under Grant no. 60974139 and the Fundamental Research Funds for the Central Universities under Grant no. 72103676 .

\section{References}

[1] S. H. Strogatz, “Exploring complex networks,” Nature, vol. 410, no. 6825, pp. 268-276, 2001.

[2] M. E. J. Newman, "The structure and function of complex networks," SIAM Review, vol. 45, no. 2, pp. 167-256, 2003.

[3] P. Wang, J. Lü, and M. J. Ogorzalek, “Global relative parameter sensitivities of the feed-forward loops in genetic networks," Neurocomputing, vol. 78, no. 1, pp. 155-165, 2012.

[4] J. Lü, X. Yu, and G. Chen, "Chaos synchronization of general complex dynamical networks," Physica A, vol. 334, no. 1-2, pp. 281-302, 2004.

[5] W. Lu and T. Chen, "New approach to synchronization analysis of linearly coupled ordinary differential systems," Physica D, vol. 213, no. 2, pp. 214-230, 2006

[6] J. Zhou, L. Xiang, and Z. Liu, "Global synchronization in general complex delayed dynamical networks and its applications," Physica A, vol. 385, no. 2, pp. 729-742, 2007.

[7] Y.-W. Wang, H. O. Wang, J.-W. Xiao, and Z.-H. Guan, "Synchronization of complex dynamical networks under recoverable attacks," Automatica, vol. 46, no. 1, pp. 197-203, 2010.

[8] S. Zheng, S. Wang, G. Dong, and Q. Bi, "Adaptive synchronization of two nonlinearly coupled complex dynamical networks with delayed coupling," Communications in Nonlinear Science and Numerical Simulation, vol. 17, no. 1, pp. 284-291, 2012.

[9] H. Peng, N. Wei, L. Li, W. Xie, and Y. Yang, "Models and synchronization of time-delayed complex dynamical networks with multi-links based on adaptive control," Physics Letters Section A, vol. 374, no. 23, pp. 2335-2339, 2010.

[10] L. Wang, H. Dai, X. Kong, and Y. Sun, "Synchronization of uncertain complex dynamical networks via adaptive control," International Journal of Robust and Nonlinear Control, vol. 19, no. 5, pp. 495-511, 2009.

[11] Z. Li, L. Jiao, and J. J. Lee, "Robust adaptive global synchronization of complex dynamical networks by adjusting time-varying coupling strength," Physica A, vol. 387, no. 5-6, pp. 1369-1380, 2008.

[12] W. Wang and J. Cao, "Synchronization in an array of linearly coupled networks with time-varying delay," Physica A, vol. 366, pp. 197-211, 2006.

[13] C. W. Wu, "Synchronization in arrays of coupled nonlinear systems with delay and nonreciprocal time-varying coupling," IEEE Transactions on Circuits and Systems II, vol. 52, no. 5, pp. 282-286, 2005.

[14] D. H. Ji, J. H. Park, W. J. Yoo, S. C. Won, and S. M. Lee, "Synchronization criterion for Lur'e type complex dynamical networks with time-varying delay," Physics Letters, Section A, vol. 374, no. 10, pp. 1218-1227, 2010.

[15] B. X. Wang and Z.-H. Guan, "Chaos synchronization in general complex dynamical networks with coupling delays," Nonlinear Analysis. Real World Applications, vol. 11, no. 3, pp. 1925-1932, 2010.

[16] H. Liu, J. Chen, J.-A. Lu, and M. Cao, "Generalized synchronization in complex dynamical networks via adaptive couplings," Physica A, vol. 389, no. 8, pp. 1759-1770, 2010.

[17] S. Liu, X. Y. Li et al., "Adaptive synchronization in complex dynamical networks with coupling delays for general graphs," Applied Mathematics and Computation. In press.

[18] L. Wang, H.-P. Dai, H. Dong, Y.-H. Shen, and Y.-X. Sun, "Adaptive synchronization of weighted complex dynamical networks with coupling time-varying delays," Physics Letters A, vol. 372, no. 20, pp. 3632-3639, 2008.

[19] X.-Y. Guo and J.-M. Li, "Projective synchronization of complex dynamical networks with time-varying coupling strength via hybrid feedback control," Chinese Physics Letters, vol. 28, no. 12, Article ID 120503, 2011.

[20] J. Lü and G. Chen, "A time-varying complex dynamical network model and its controlled synchronization criteria," IEEE Transactions on Automatic Control, vol. 50, no. 6, pp. 841-846, 2005.

[21] J. Zhou, J.-a. Lu, and J. Lü, "Pinning adaptive synchronization of a general complex dynamical network," Automatica, vol. 44, no. 4, pp. 996-1003, 2008.

[22] J. Zhou, J.-a. Lu, and J. Lü, "Adaptive synchronization of an uncertain complex dynamical network," IEEE Transactions on Automatic Control, vol. 51, no. 4, pp. 652-656, 2006. 
[23] W.-X. Wang, J. Lü, G. Chen, and P. M. Hui, "Phase transition and hysteresis loop in structured games with global updating," Physical Review E, vol. 77, no. 4, Article ID 046109, 2008.

[24] J.-X. Xu and Y. Tan, "A composite energy function-based learning control approach for nonlinear systems with time-varying parametric uncertainties," IEEE Transactions on Automatic Control, vol. 47, no. 11, pp. 1940-1945, 2002.

[25] W. Lin and C. Qian, "Adaptive control of nonlinearly parameterized systems: the smooth feedback case," IEEE Transactions on Automatic Control, vol. 47, no. 8, pp. 1249-1266, 2002.

[26] J.-M. Li, Y.-L. Wang, and X.-M. Li, "Adaptive iterative learning control for nonlinear parameterizedsystems with unknown time-varying delays," Control Theory and Applications, vol. 28, no. 6, pp. 861868, 2011.

[27] Y. Chen, J. Lü, F. Han, and X. Yu, "On the cluster consensus of discrete-time multi-agent systems," Systems $\mathcal{E}$ Control Letters, vol. 60, no. 7, pp. 517-523, 2011. 


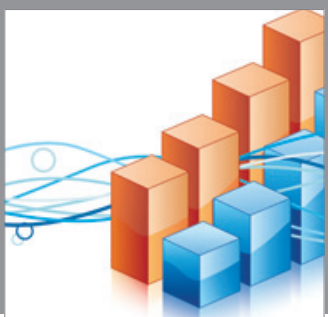

Advances in

Operations Research

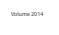

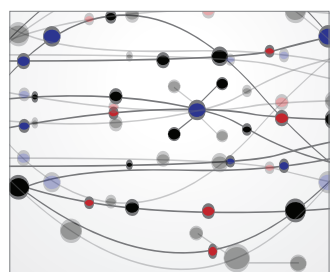

\section{The Scientific} World Journal
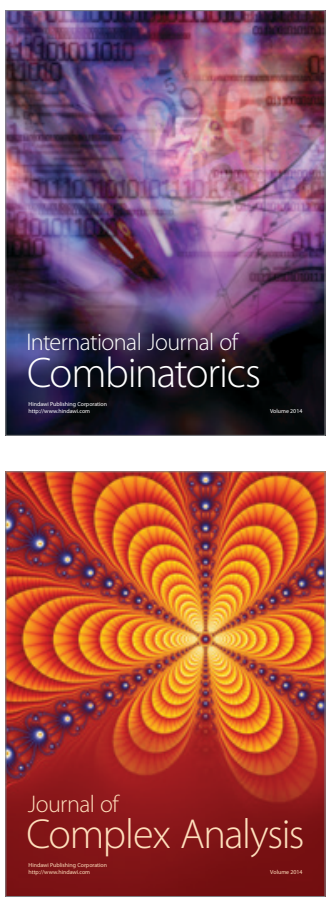

International Journal of

Mathematics and

Mathematical

Sciences
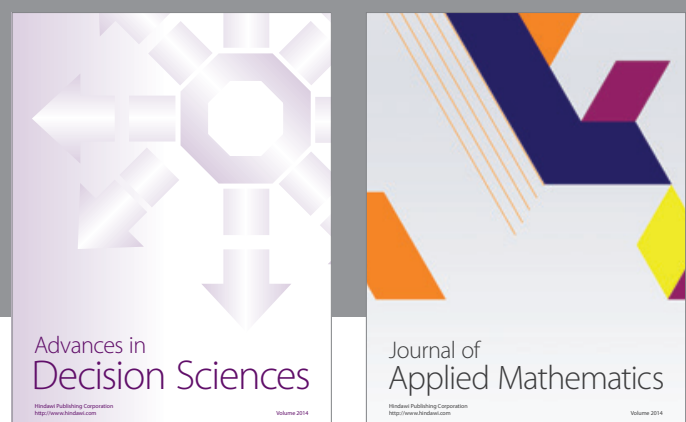

Journal of

Applied Mathematics
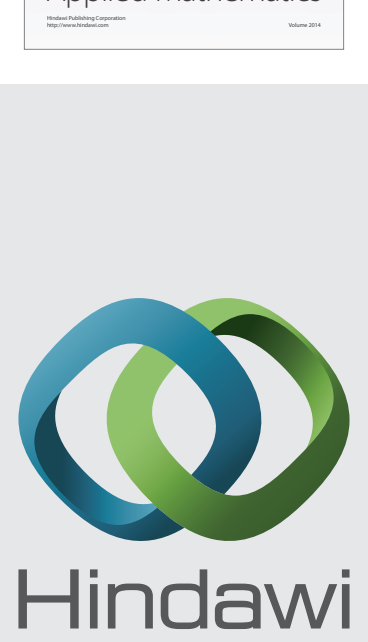

Submit your manuscripts at http://www.hindawi.com
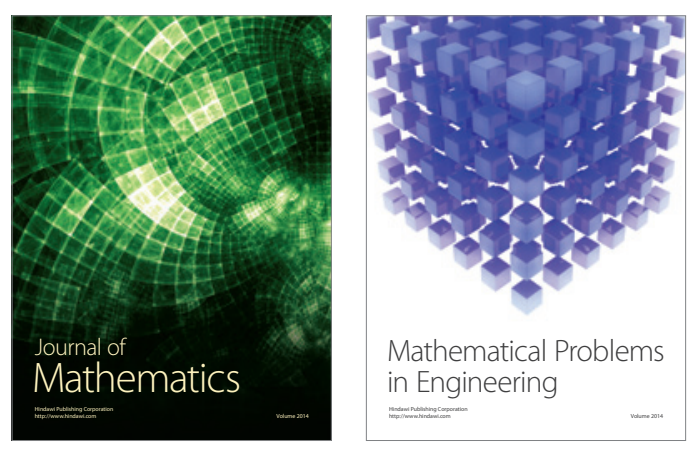

Mathematical Problems in Engineering
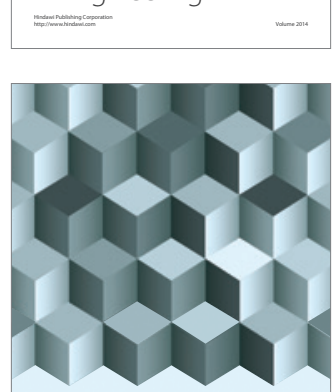

Journal of

Function Spaces
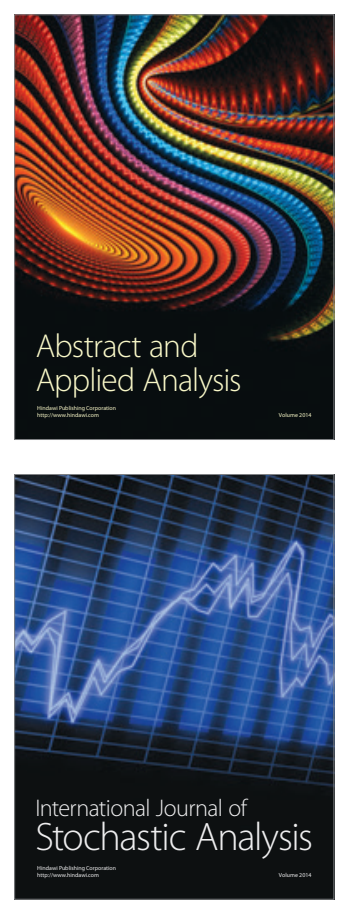

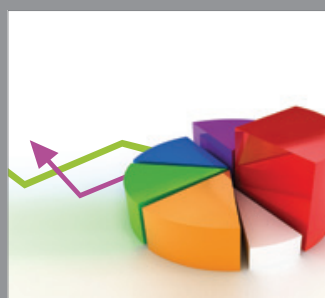

ournal of

Probability and Statistics

Promensencen
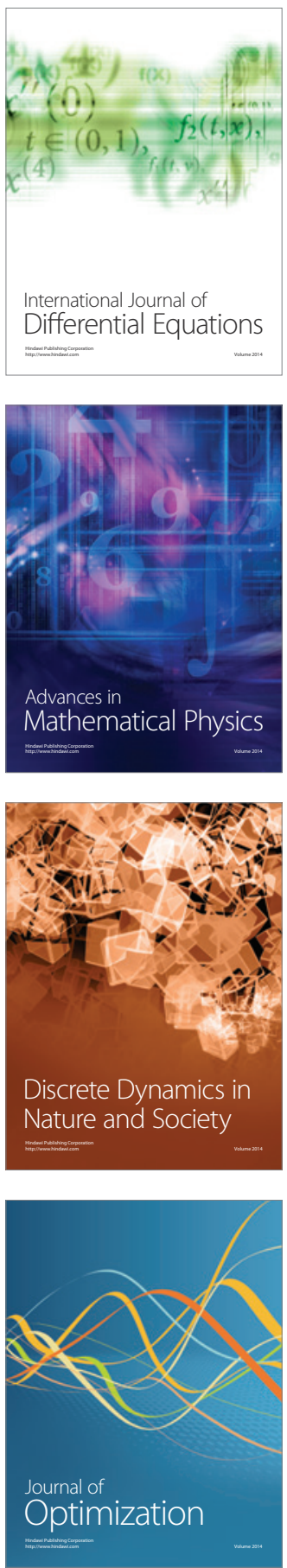